\title{
Synthesis, Characterization and Antimicrobial Activity of New Thiadiazole Derivatives
}

\author{
Pooja Mullick, Suroor A. Khan, Surajpal Verma, and Ozair Alam.
}

\author{
Department of Pharmaceutical Chemistry, Faculty of Pharmacy, Jamia Hamdard (Hamdard University) Hamdard Nagar, \\ New Delhi-110062, India. EE-mail: poojamullick7@rediffmail.com \\ Received December 2, 2009, Accepted February 16, 2010
}

\begin{abstract}
A series of thiadiazole derivatives were synthesized with differently substituted benzoic acids which were cyclized to give differently substituted thiazolidin-4-one. Elemental analysis, IR, ${ }^{1} \mathrm{H}$ NMR, ${ }^{13} \mathrm{C}$ NMR and mass spectral data confirmed the structure of the newly synthesized compounds. The derivatives of these moieties were evaluated for antimicrobial activity. Most of the synthesized compounds showed good antimicrobial activity at 200 and $100 \mu \mathrm{g} / \mathrm{mL}$. Compounds showed most significant antibacterial activity against gram negative test organism Escherichia coli and most significant antifungal activity against test organisms Aspergillus niger and Candida albicans. It was observed that compounds with $\mathrm{OCH}_{3}$ at 3 , 4 position of phenyl ring [5(a-l)] were more potent against microbes as compared to compounds having unsubstituted phenyl ring $[\mathbf{4}(\mathbf{a}-\mathbf{I})]$.
\end{abstract}

Key Words: Thiadiazole, Thiazolidin-4-one, Antimicrobial activity

\section{Introduction}

Widespread antibiotic resistance, the emergence of new pathogens in addition to the resurgence of old ones, and the lack of effective new therapeutics exacerbate the problems of antimicrobial resistance. ${ }^{1}$

The recent literature is enriched with progressive findings about the synthesis of 1,3,4-thiadiazole moiety and their broad spectrum of pharmacological actions such as anti-bacterial, ${ }^{2}$ anti-fungal, ${ }^{2}$ anti-tubercular, ${ }^{3}$ anti-convulsant, ${ }^{4}$ anti-inflammatory, ${ }^{5}$ analgesic, ${ }^{5}$ anti-anxiety, anti-depressant ${ }^{6}$ and anti-viral. ${ }^{7}$ Thiazolidine-4-one moiety also found to possess diverse biological activities such as anti-microbial ${ }^{8}$, anti-convulsant, ${ }^{9}$ antidiarrheal, ${ }^{10}$ anti-cancer, ${ }^{11} \mathrm{~K}^{+}$channel inhibitory, ${ }^{12}$ anti-histaminic. ${ }^{13}$ These two heterocyclic moieties individually showed potent pharmacological activity especially antimicrobial and thus aroused our interest in synthesizing the combination of the two moieties. Hence, the present paper is focused on synthesis of thiazolidineone in combination with thiadiazole to enhance their antimicrobial properties.

\section{Experimental}

General method for synthesis of 2-amino-5-(substituted phenyl)-(1,3,4)-thiadiazole 1(a-l). A mixture of $40 \mathrm{mmol}$ of differently substituted benzoic acids and the equimolar amount of thiosemicarbazide and phosphorous oxychloride $(30 \mathrm{~mL})$ was refluxed gently for 2 - $4 \mathrm{hrs}$. The reaction mixture was allowed to cool; ice cold water $(100 \mathrm{~mL})$ was added to the flask. The mixture was again set for refluxing for about $4 \mathrm{hrs}$. and filtered. The solution was neutralized with ammonia solution. The precipitate was filtered, washed with water, dried and recrystallized from ethanol-water to yield compounds $\mathbf{1}(\mathbf{a}-\mathbf{l})$. The purity of compounds was analyzed by TLC using benzene: acetone (9:1) as mobile phase.

2-Amino-5-phenyl-(1,3,4)-thiadiazole 1(a): $\mathrm{mp} 224$ - $225^{\circ} \mathrm{C}$; \%Yield: 71; IR (KBr) cm ${ }^{-1}$ : $3496(\mathrm{NH}), 1595(\mathrm{C}=\mathrm{N}), 1604$ $(\mathrm{C}=\mathrm{C}) ;{ }^{1} \mathrm{H}$ NMR $\left(300 \mathrm{MHz}, \mathrm{DMSO}-d_{6}\right) \delta$ 6.64-7.19 (m, 5H,
ArH), 12.04 (s, 2H, $\left.\mathrm{NH}_{2}\right)$.

2-Amino-5-(2-methylphenyl)-(1,3,4)-thiadiazole 1(b): mp 210 - $211^{\circ} \mathrm{C}$; \%Yield: 73; IR (KBr) cm ${ }^{-1}: 3446(\mathrm{NH}), 1593(\mathrm{C}=$ $\mathrm{N}), 1636(\mathrm{C}=\mathrm{C}) ;{ }^{1} \mathrm{H}$ NMR $\left(300 \mathrm{MHz}, \mathrm{DMSO}-d_{6}\right) \delta 2.33(\mathrm{~s}, 3 \mathrm{H}$, $\left.\mathrm{CH}_{3}\right), 6.64-7.19$ (m, 4H, ArH), 12.04 (s, 2H, NH$)_{2}$.

2-amino-5-(4-methylphenyl)-(1,3,4)-thiadiazole 1(c): $\mathrm{mp}$ 215 - $216^{\circ} \mathrm{C}$; \% Yield: 79; IR ( $\left.\mathrm{KBr}\right) \mathrm{cm}^{-1}$ : $3477\left(\mathrm{NH}_{2}\right), 1587$ $(\mathrm{C}=\mathrm{N}), 1644(\mathrm{C}=\mathrm{C}) ;{ }^{1} \mathrm{H}$ NMR $\left(300 \mathrm{MHz}, \mathrm{DMSO}-d_{6}\right) \delta 2.35(\mathrm{~s}$, $\left.3 \mathrm{H}, \mathrm{CH}_{3}\right), 6.71-7.20$ (m, 4H, $\left.\mathrm{ArH}\right), 12.15$ (s, 2H, $\mathrm{NH}_{2}$ ).

2-Amino-5-(2-chlorophenyl)-(1,3,4)-thiadiazole 1(d): $\mathrm{mp}$ 204 - $206{ }^{\circ} \mathrm{C}$; \% Yield: 67; IR (KBr) cm ${ }^{-1}: 3465(\mathrm{NH}), 1557$ $(\mathrm{C}=\mathrm{N}), 1614(\mathrm{C}=\mathrm{C}) ;{ }^{1} \mathrm{H}$ NMR $\left(300 \mathrm{MHz}, \mathrm{DMSO}-d_{6}\right) \delta 6.78-$ 7.24 (m, 4H, ArH), 12.04 (s, 2H, $\mathrm{NH}_{2}$ ).

2-Amino-5-(4-chlorophenyl)-(1,3,4)-thiadiazole 1(e): mp 230 - $232{ }^{\circ} \mathrm{C}$; \% Yield: 77; IR (KBr) cm ${ }^{-1}: 3468(\mathrm{NH}), 1572$ $(\mathrm{C}=\mathrm{N}), 1594(\mathrm{C}=\mathrm{C}) ;{ }^{1} \mathrm{H}$ NMR $\left(300 \mathrm{MHz}, \mathrm{DMSO}-d_{6}\right) \delta 6.86-$ 7.25 (m, 4H, ArH), 11.64 (s, 2H, $\mathrm{NH}_{2}$ ).

2-Amino-5-(2-bromophenyl)-(1,3,4)-thiadiazole 1(f): $\mathrm{mp}$ 192 - $194{ }^{\circ} \mathrm{C}$; \% Yield: 62; IR (KBr) cm ${ }^{-1}: 3492(\mathrm{NH}), 1583$ $(\mathrm{C}=\mathrm{N}), 1619(\mathrm{C}=\mathrm{C}) ;{ }^{1} \mathrm{H}$ NMR $\left(300 \mathrm{MHz}, \mathrm{DMSO}-d_{6}\right) \delta 6.84-$ 7.17 (m, 4H, ArH), 12.16 (s, 2H, $\mathrm{NH}_{2}$ ).

2-Amino-5-(4-bromophenyl)-(1,3,4)-thiadiazole 1(g): $\mathrm{mp}$ $247-248{ }^{\circ} \mathrm{C}$; \% Yield: 61; IR (KBr) cm ${ }^{-1}: 3396(\mathrm{NH}), 1569$ $(\mathrm{C}=\mathrm{N}), 1582(\mathrm{C}=\mathrm{C}) ;{ }^{1} \mathrm{H}$ NMR $\left(300 \mathrm{MHz}, \mathrm{DMSO}-d_{6}\right) \delta 6.64-$ 7.19 (m, 4H, ArH), 12.07 (s, 2H, $\mathrm{NH}_{2}$ ).

2-Amino-5-(3-nitrophenyl)-(1,3,4)-thiadiazole 1(h): $\mathrm{mp} 236$ $238^{\circ} \mathrm{C}$; \% Yield: 66; IR (KBr) cm $\mathrm{cm}^{-1}: 3426(\mathrm{NH}), 1564(\mathrm{C}=\mathrm{N})$, $1558(\mathrm{C}=\mathrm{C}) ;{ }^{1} \mathrm{H}$ NMR $\left(300 \mathrm{MHz}, \mathrm{DMSO}-d_{6}\right) \delta 6.88-7.23(\mathrm{~m}$, $4 \mathrm{H}, \mathrm{ArH}), 12.24\left(\mathrm{~s}, 2 \mathrm{H}, \mathrm{NH}_{2}\right)$.

2-Amino-5-(4-nitrophenyl)-(1,3,4)-thiadiazole 1(i): $\mathrm{mp} 227$ $229^{\circ} \mathrm{C}$; \%Yield: 60; IR (KBr) cm $\mathrm{cm}^{-1}: 3452(\mathrm{NH}), 1536(\mathrm{C}=\mathrm{N})$, $1548(\mathrm{C}=\mathrm{C}) ;{ }^{1} \mathrm{H}$ NMR $\left(300 \mathrm{MHz}, \mathrm{DMSO}-d_{6}\right) \delta 6.88-7.24(\mathrm{~m}$, $4 \mathrm{H}, \mathrm{ArH}), 12.35$ (s, 2H, $\mathrm{NH}_{2}$ ).

2-Amino-5-(2,4-dichlorophenyl)-(1,3,4)-thiadiazole 1(j): $\mathrm{mp}$ 240 - $242{ }^{\circ} \mathrm{C}$; \% Yield: 82; IR (KBr) cm ${ }^{-1}: 3506(\mathrm{NH}), 1598$ $(\mathrm{C}=\mathrm{N}), 1579(\mathrm{C}=\mathrm{C}) ;{ }^{1} \mathrm{H}$ NMR $\left(300 \mathrm{MHz}, \mathrm{DMSO}-d_{6}\right) \delta 7.14-$ 7.30 (m, 3H, ArH), 12.34 (s, 2H, $\mathrm{NH}_{2}$ ).

2-Amino-5-(2-hydroxyphenyl)-(1,3,4)-thiadiazole 1(k): mp 
184 - $185{ }^{\circ} \mathrm{C}$; \%Yield: 73; IR (KBr) $\mathrm{cm}^{-1}: 3493(\mathrm{NH}), 1575$ $(\mathrm{C}=\mathrm{N}), 1549(\mathrm{C}=\mathrm{C}) ;{ }^{1} \mathrm{H}$ NMR $\left(300 \mathrm{MHz}, \mathrm{DMSO}-d_{6}\right) \delta 6.58-6.93$ (m, 4H, ArH), 9.42 (s, 1H, OH), 11.56 (s, 2H, $\mathrm{NH}_{2}$ ).

2-Amino-5-(4-methoxyphenyl)-(1,3,4)-thiadiazole 1(l): $\mathrm{mp}$ 209 - $210{ }^{\circ} \mathrm{C}$; \% Yield: 78; IR (KBr) cm ${ }^{-1}: 3426(\mathrm{NH}), 1555$ $(\mathrm{C}=\mathrm{N}), 1618(\mathrm{C}=\mathrm{C}) ;{ }^{1} \mathrm{H}$ NMR $\left(300 \mathrm{MHz}, \mathrm{DMSO}-d_{6}\right) \delta 3.16$ (s, 3H, $\left.\mathrm{OCH}_{3}\right), 6.64-7.19$ (m, 4H, ArH), 11.46 (s, 2H, $\mathrm{NH}_{2}$ ).

General method for synthesis of 2-chloro- $\mathrm{N}$-[5-(substituted phenyl)-[1,3,4]-thiadiazol-2-yl]-acetamide 2(a-l). To the mixture of appropriately substituted compound $\mathbf{1}(\mathbf{a}-\mathbf{l})(10 \mathrm{mmol})$ in $15 \mathrm{~mL}$ of dry benzene and $2 \mathrm{~mL}$ of dry pyridine, chloroacetylchloride $(20 \mathrm{mmol})$ in $10 \mathrm{~mL}$ of dry benzene was added drop wise with a constant stirring at room temperature. After complete addition, the reaction mixture was refluxed for about 6 - 8 hrs. and poured over crushed ice. The precipitate was filtered, washed with water, dried and recrystallized from dioxane-water to yield compound 2(a-l). The purity of compounds was analyzed by TLC using benzene: acetone (9:1) as mobile phase.

2-Chloro- $N$-[5-phenyl-[1,3,4]-thiadiazol-2-yl]-acetamide 2(a): $\mathrm{mp} 228$ - $229^{\circ} \mathrm{C}$; \% Yield: 87; IR (KBr) cm ${ }^{-1}: 3130(\mathrm{NH})$, $1695(\mathrm{C}=\mathrm{O}), 1572(\mathrm{C}=\mathrm{N}), 764(\mathrm{C}-\mathrm{Cl}) ;{ }^{1} \mathrm{H}$ NMR $(300 \mathrm{MHz}$, DMSO- $\left.d_{6}\right) \delta 4.48\left(\mathrm{~s}, 2 \mathrm{H}, \mathrm{CH}_{2}\right), 7.54-7.96(\mathrm{~m}, 5 \mathrm{H}, \mathrm{ArH}), 13.07$ (bs, $1 \mathrm{H}, \mathrm{NH}) ;{ }^{13} \mathrm{C}$ NMR $\left(75 \mathrm{MHz}, \mathrm{DMSO}-d_{6}\right) \delta 48.6,126.7$, 127.1, 128.6, 129.0, 129.4, 136.5, 154.2, 161.5, 164.2; FAB-MS $m / z: 254(\mathrm{M})^{+}, 256(\mathrm{M}+2)^{+}$.

2-Chloro- $\mathrm{N}$-[5-(2-methylphenyl)-[1,3,4]-thiadiazol-2-yl]acetamide 2(b): $\mathrm{mp} 213-215^{\circ} \mathrm{C}$; \% Yield: 82; IR (KBr) $\mathrm{cm}^{-1}$ : $3340(\mathrm{NH}), 1648(\mathrm{C}=\mathrm{O}), 1552(\mathrm{C}=\mathrm{N}), 761(\mathrm{C}-\mathrm{Cl}) ;{ }^{1} \mathrm{H}$ NMR $\left(300 \mathrm{MHz}, \mathrm{DMSO}-d_{6}\right) \delta 2.34\left(\mathrm{~s}, 3 \mathrm{H}, \mathrm{CH}_{3}\right), 4.28\left(\mathrm{~s}, 2 \mathrm{H}, \mathrm{CH}_{2}\right)$, 7.52-7.87 (m, 4H, ArH), 12.94 (bs, 1H, NH).

2-Chloro- $\mathrm{N}$-[5-(4-methylphenyl)-[1,3,4]-thiadiazol-2-yl]acetamide 2(c): $\mathrm{mp} 238-240{ }^{\circ} \mathrm{C}$; \% Yield: 87 ; IR $(\mathrm{KBr}) \mathrm{cm}^{-1}$ : $3160(\mathrm{NH}), 1653(\mathrm{C}=\mathrm{O}), 1574(\mathrm{C}=\mathrm{N}), 766(\mathrm{C}-\mathrm{Cl}) ;{ }^{1} \mathrm{H}$ NMR (300 MHz, DMSO- $\left.d_{6}\right) \delta 2.36\left(\mathrm{~s}, 3 \mathrm{H}, \mathrm{CH}_{3}\right), 4.31\left(\mathrm{~s}, 2 \mathrm{H}, \mathrm{CH}_{2}\right)$, 7.53-7.84 (m, 4H, ArH), 12.64 (bs, 1H, NH).

2-Chloro- $\mathrm{N}$-[5-(2-chlorophenyl)-[1,3,4]-thiadiazol-2-yl]acetamide 2(d): $\mathrm{mp} 229-230^{\circ} \mathrm{C}$; \% Yield: 86; IR (KBr) $\mathrm{cm}^{-1}$ : $3364(\mathrm{NH}), 1687(\mathrm{C}=\mathrm{O}), 1535(\mathrm{C}=\mathrm{N}), 757(\mathrm{C}-\mathrm{Cl}) ;{ }^{1} \mathrm{H}$ NMR (300 MHz, DMSO- $\left.d_{6}\right) \delta 4.29$ (s, 2H, $\left.\mathrm{CH}_{2}\right), 7.58-7.91(\mathrm{~m}, 4 \mathrm{H}$, ArH), 12.65 (bs, 1H, NH).

2-Chloro- $\mathrm{N}$-[5-(4-chlorophenyl)-[1,3,4]-thiadiazol-2-yl]acetamide 2(e): $\mathrm{mp} 243-244^{\circ} \mathrm{C}$; \% Y ield: 90; IR (KBr) $\mathrm{cm}^{-1}$ : $3230(\mathrm{NH}), 1686(\mathrm{C}=\mathrm{O}), 1554(\mathrm{C}=\mathrm{N}), 759(\mathrm{C}-\mathrm{Cl}) ;{ }^{1} \mathrm{H}$ NMR $\left(300 \mathrm{MHz}, \mathrm{DMSO}-d_{6}\right) \delta 4.32\left(\mathrm{~s}, 2 \mathrm{H}, \mathrm{CH}_{2}\right), 7.53-7.87$ (m, 4H, $\mathrm{ArH}), 13.18$ (bs, 1H, NH).

2-Chloro- $\boldsymbol{N}$-[5-(2-bromophenyl)-[1,3,4]-thiadiazol-2-yl]acetamide 2(f): $\mathrm{mp} 263-265^{\circ} \mathrm{C}$; \% Yield: 69 ; IR (KBr) $\mathrm{cm}^{-1}$ : $3275(\mathrm{NH}), 1646(\mathrm{C}=\mathrm{O}), 1552(\mathrm{C}=\mathrm{N}), 754(\mathrm{C}-\mathrm{Cl}) ;{ }^{1} \mathrm{H}$ NMR (300 MHz, DMSO- $\left.d_{6}\right) \delta 4.37\left(\mathrm{~s}, 2 \mathrm{H}, \mathrm{CH}_{2}\right), 7.52-7.84(\mathrm{~m}, 4 \mathrm{H}$, ArH), 12.73 (bs, 1H, NH).

2-Chloro- $\mathrm{N}$-[5-(4-bromophenyl)-[1,3,4]-thiadiazol-2-yl]acetamide 2(g): $\mathrm{mp} 241-242{ }^{\circ} \mathrm{C}$; \% Yield: 63; IR (KBr) $\mathrm{cm}^{-1}$ : $3264(\mathrm{NH}), 1665(\mathrm{C}=\mathrm{O}), 1567(\mathrm{C}=\mathrm{N}), 762(\mathrm{C}-\mathrm{Cl}) ;{ }^{1} \mathrm{H}$ NMR (300 MHz, DMSO- $\left.d_{6}\right) \delta 4.42\left(\mathrm{~s}, 2 \mathrm{H}, \mathrm{CH}_{2}\right), 7.67-7.91(\mathrm{~m}, 4 \mathrm{H}$, ArH), 11.86 (bs, 1H, NH).

2-Chloro- $N$-[5-(3-nitrophenyl)-[1,3,4]-thiadiazol-2-yl]-acetamide 2(h): $\mathrm{mp} 168-170^{\circ} \mathrm{C}$; \%Yield: 64; IR (KBr) cm ${ }^{-1}: 3374$ $(\mathrm{NH}), 1673(\mathrm{C}=\mathrm{O}), 1577(\mathrm{C}=\mathrm{N}), 751(\mathrm{C}-\mathrm{Cl}) ;{ }^{1} \mathrm{H}$ NMR $(300$
MHz, DMSO- $\left.d_{6}\right) \delta 4.27$ (s, 2H, $\left.\mathrm{CH}_{2}\right), 7.58-7.82(\mathrm{~m}, 4 \mathrm{H}, \mathrm{ArH})$, 12.74 (bs, $1 \mathrm{H}, \mathrm{NH})$.

2-Chloro- $\mathbf{N}$-[5-(4-nitrophenyl)-[1,3,4]-thiadiazol-2-yl]-acetamide 2(i): $\mathrm{mp} 162-163^{\circ} \mathrm{C}$; \% Yield: 67 ; IR (KBr) cm ${ }^{-1}: 3266$ $(\mathrm{NH}), 1646(\mathrm{C}=\mathrm{O}), 1554(\mathrm{C}=\mathrm{N}), 755(\mathrm{C}-\mathrm{Cl}) ;{ }^{1} \mathrm{H}$ NMR $(300$ MHz, DMSO- $\left.d_{6}\right) \delta 4.25$ (s, 2H, $\left.\mathrm{CH}_{2}\right), 7.54-7.81$ (m, 4H, ArH), 11.53 (bs, $1 \mathrm{H}, \mathrm{NH}$ ).

2-Chloro- $\mathrm{N}$-[5-(2,4-dichlorophenyl)-[1,3,4]-thiadiazol-2yll-acetamide 2(j): $\mathrm{mp} 246-247^{\circ} \mathrm{C}$; \% Yield: 82 ; IR (KBr) cm ${ }^{-1}$ : $3276(\mathrm{NH}), 1657(\mathrm{C}=\mathrm{O}), 1576(\mathrm{C}=\mathrm{N}), 759(\mathrm{C}-\mathrm{Cl}) ;{ }^{1} \mathrm{H}$ NMR $\left(300 \mathrm{MHz}, \mathrm{DMSO}-d_{6}\right) \delta 4.46\left(\mathrm{~s}, 2 \mathrm{H}, \mathrm{CH}_{2}\right), 7.63-7.79(\mathrm{~m}, 3 \mathrm{H}$, ArH), 12.57 (bs, 1H, NH).

2-Chloro- $N$-[5-(2-hydroxyphenyl)-[1,3,4]-thiadiazol-2-yl]acetamide 2(k): $\mathrm{mp} 203-205^{\circ} \mathrm{C}$; \% Yield: 79; IR (KBr) $\mathrm{cm}^{-1}$ : $3267(\mathrm{NH}), 1583(\mathrm{C}=\mathrm{O}), 1524(\mathrm{C}=\mathrm{N}), 751(\mathrm{C}-\mathrm{Cl}) ;{ }^{1} \mathrm{H}$ NMR $\left(300 \mathrm{MHz}, \mathrm{DMSO}-d_{6}\right) \delta 4.23\left(\mathrm{~s}, 2 \mathrm{H}, \mathrm{CH}_{2}\right), 7.43-7.66(\mathrm{~m}, 4 \mathrm{H}$, ArH), 9.47 (s, 1H, OH), 12.37 (bs, 1H, NH).

2-Chloro- $\mathrm{N}$-[5-(4-methoxyphenyl)-[1,3,4]-thiadiazol-2-yl]acetamide 2(I): $\mathrm{mp} 231-232^{\circ} \mathrm{C}$; \% Yield: 87 ; IR (KBr) $\mathrm{cm}^{-1}$ : $3370(\mathrm{NH}), 1637(\mathrm{C}=\mathrm{O}), 1576(\mathrm{C}=\mathrm{N}), 757(\mathrm{C}-\mathrm{Cl}) ;{ }^{1} \mathrm{H}$ NMR $\left(300 \mathrm{MHz}, \mathrm{DMSO}-d_{6}\right) \delta 3.43\left(\mathrm{~s}, 3 \mathrm{H}, \mathrm{OCH}_{3}\right), 4.26\left(\mathrm{~s}, 2 \mathrm{H}, \mathrm{CH}_{2}\right)$, 7.52-7.71 (m, 4H, ArH), 11.50 (bs, 1H, NH).

General method for synthesis of 2-[\{5-(substituted phenyl)[1,3,4]-thiadiazol-2-yl imino]-1,3-thiazolidin-4-one 3(a-l). 7 mmol of each compound $\mathbf{2}(\mathbf{a}-\mathbf{I})$ and ammonium thiocyanate $(15 \mathrm{mmol})$ in $35 \mathrm{~mL}$ ethanol was refluxed for $3 \mathrm{hrs}$, hold reaction mixture overnight. The product obtained was filtered, dried and recrystallized from ethanol-water to yield compound 3(a-l). The purity of compounds was analyzed by TLC using benzene: acetone $(9: 1)$ as mobile phase.

2-[\{5-Phenyl-[1,3,4]-thiadiazol-2-yl\} imino]-1,3-thiazolidin4-one 3(a): $\mathrm{mp} 128-130{ }^{\circ} \mathrm{C}$; \% Yield: 76; IR (KBr) cm ${ }^{-1}$ : 3374 $(\mathrm{NH}), 1762(\mathrm{C}=\mathrm{O}), 1592(\mathrm{C}=\mathrm{N})$; ${ }^{1} \mathrm{H}$ NMR $(300 \mathrm{MHz}$, DMSO$\left.d_{6}\right) \delta 4.13\left(\mathrm{~s}, 2 \mathrm{H}, \mathrm{CH}_{2}-\mathrm{CO}\right), 7.52-7.75(\mathrm{~m}, 5 \mathrm{H}, \mathrm{ArH}), 12.38(\mathrm{bs}$, $1 \mathrm{H}, \mathrm{NH}, \mathrm{D}_{2} \mathrm{O}$-exchangeble); ${ }^{13} \mathrm{C} \mathrm{NMR}\left(75 \mathrm{MHz}, \mathrm{DMSO}-d_{6}\right) \delta$ 37.4, 126.4, 127.7, 128.3, 129.0, 129.5, 136.7, 154.1, 162.6, 163.8, 173.4; FAB-MS $m / z: 276(\mathrm{M})^{+}$

2-[\{5-(2-Methylphenyl)-[1,3,4]-thiadiazol-2-yl\} imino]-1,3thiazolidin-4-one 3(b): $\mathrm{mp} 133$ - $135^{\circ} \mathrm{C}$; \% Yield: 72; IR (KBr) $\mathrm{cm}^{-1}$ : $3385(\mathrm{NH}), 1775(\mathrm{C}=\mathrm{O}), 1575(\mathrm{C}=\mathrm{N}) ;{ }^{1} \mathrm{H}$ NMR (300 $\left.\mathrm{MHz}, \mathrm{DMSO}-d_{6}\right) \delta 2.36\left(\mathrm{~s}, 3 \mathrm{H}, \mathrm{CH}_{3}\right), 4.08$ (s, 2H, $\left.\mathrm{CH}_{2}-\mathrm{CO}\right)$, 7.42-7.58 (m, 4H, ArH), 12.18 (bs, 1H, NH).

2-[\{5-(4-Methylphenyl)-[1,3,4]-thiadiazol-2-yl\} imino]-1,3thiazolidin-4-one 3(c): mp $122-123{ }^{\circ} \mathrm{C}$; \% Yield: 83; IR (KBr) $\mathrm{cm}^{-1}$ : $3402(\mathrm{NH}), 1757(\mathrm{C}=\mathrm{O}), 1587(\mathrm{C}=\mathrm{N}) ;{ }^{1} \mathrm{H}$ NMR $(300$ $\left.\mathrm{MHz}, \mathrm{DMSO}-d_{6}\right) \delta 2.32\left(\mathrm{~s}, 3 \mathrm{H}, \mathrm{CH}_{3}\right), 4.13\left(\mathrm{~s}, 2 \mathrm{H}, \mathrm{CH}_{2}-\mathrm{CO}\right)$, 7.49-7.63 (m, 4H, ArH), 11.85 (bs, 1H, NH).

2-[\{5-(2-Chlorophenyl)-[1,3,4]-thiadiazol-2-yl\} imino]-1,3thiazolidin-4-one 3(d): $\mathrm{mp} 162-164{ }^{\circ} \mathrm{C}$; \% Yield: 71; IR (KBr) $\mathrm{cm}^{-1}$ : $3418(\mathrm{NH}), 1735(\mathrm{C}=\mathrm{O}), 1592(\mathrm{C}=\mathrm{N}) ;{ }^{1} \mathrm{H}$ NMR (300 $\left.\mathrm{MHz}, \mathrm{DMSO}-d_{6}\right) \delta 4.51$ (s, 2H, $\left.\mathrm{CH}_{2}-\mathrm{CO}\right), 7.61-7.74$ (m, $4 \mathrm{H}$, ArH), 13.08 (bs, 1H, NH).

2-[\{5-(4-Chlorophenyl)-[1,3,4]-thiadiazol-2-yl\} imino]-1,3thiazolidin-4-one 3(e): mp $174-177^{\circ} \mathrm{C}$; \%Yield: 78; IR (KBr) $\mathrm{cm}^{-1}$ : $3428(\mathrm{NH}), 1751(\mathrm{C}=\mathrm{O}), 1593(\mathrm{C}=\mathrm{N}) ;{ }^{1} \mathrm{H}$ NMR (300 $\left.\mathrm{MHz}, \mathrm{DMSO}-d_{6}\right) \delta 3.97$ (s, 2H, $\left.\mathrm{CH}_{2}-\mathrm{CO}\right), 7.63-7.78$ (m, 4H, ArH), 12.86 (bs, 1H, NH).

2-[\{5-(2-Bromophenyl)-[1,3,4]-thiadiazol-2-yl\} imino]-1,3- 
thiazolidin-4-one 3(f): $\mathrm{mp} 117-119^{\circ} \mathrm{C}$; \% Yield: 69; IR (KBr) $\mathrm{cm}^{-1}$ : $3391(\mathrm{NH}), 1695(\mathrm{C}=\mathrm{O}), 1587(\mathrm{C}=\mathrm{N}) ;{ }^{1} \mathrm{H}$ NMR $(300$ $\left.\mathrm{MHz}, \mathrm{DMSO}-d_{6}\right) \delta 4.31$ (s, 2H, $\left.\mathrm{CH}_{2}-\mathrm{CO}\right), 7.63-7.77$ (m, 4H, ArH), 12.89 (bs, 1H, NH).

2-[\{5-(4-Bromophenyl)-[1,3,4]-thiadiazol-2-yl\} imino]-1,3thiazolidin-4-one 3(g): $\mathrm{mp} 135-136{ }^{\circ} \mathrm{C}$; \% Yield: 64; IR (KBr) $\mathrm{cm}^{-1}$ : $3386(\mathrm{NH}), 1705(\mathrm{C}=\mathrm{O}), 1582(\mathrm{C}=\mathrm{N}) ;{ }^{1} \mathrm{H}$ NMR $(300$ $\left.\mathrm{MHz}, \mathrm{DMSO}-d_{6}\right) \delta 4.27$ (s, 2H, $\left.\mathrm{CH}_{2}-\mathrm{CO}\right), 7.66-7.83(\mathrm{~m}, 4 \mathrm{H}$, ArH), 11.93 (bs, 1H, NH).

2-[\{5-(3-Nitrophenyl)-[1,3,4]-thiadiazol-2-yl\} imino]-1,3thiazolidin-4-one 3(h): $\mathrm{mp} 107-110^{\circ} \mathrm{C}$; \% Yield: 54; IR (KBr) $\mathrm{cm}^{-1}$ : $3372(\mathrm{NH}), 1696(\mathrm{C}=\mathrm{O}), 1597(\mathrm{C}=\mathrm{N}) ;{ }^{1} \mathrm{H}$ NMR $(300$ $\left.\mathrm{MHz}, \mathrm{DMSO}-d_{6}\right) \delta 4.11\left(\mathrm{~s}, 2 \mathrm{H}, \mathrm{CH}_{2}-\mathrm{CO}\right), 7.37-7.53(\mathrm{~m}, 4 \mathrm{H}$, ArH), 13.03 (bs, 1H, NH).

2-[\{5-(4-Nitrophenyl)-[1,3,4]-thiadiazol-2-yl\} imino]-1,3thiazolidin-4-one 3(i): $\mathrm{mp} 146-148^{\circ} \mathrm{C}$; \%Yield: 57; IR (KBr) $\mathrm{cm}^{-1}$ : $3418(\mathrm{NH}), 1735(\mathrm{C}=\mathrm{O}), 1592(\mathrm{C}=\mathrm{N}) ;{ }^{1} \mathrm{H}$ NMR $(300$ $\left.\mathrm{MHz}, \mathrm{DMSO}-d_{6}\right) \delta 3.84$ (s, 2H, $\left.\mathrm{CH}_{2}-\mathrm{CO}\right), 7.37-7.54(\mathrm{~m}, 4 \mathrm{H}$, $\mathrm{ArH}), 12.73$ (bs, 1H, NH).

2-[\{5-(2,4-Dichlorophenyl)-[1,3,4]-thiadiazol-2-yl $\}$ imino]1,3-thiazolidin-4-one 3(j): mp $186-188^{\circ} \mathrm{C}$; \% Yield: 70; IR $(\mathrm{KBr}) \mathrm{cm}^{-1}$ : $3425(\mathrm{NH}), 1684(\mathrm{C}=\mathrm{O}), 1583(\mathrm{C}=\mathrm{N}) ;{ }^{1} \mathrm{H}$ NMR $\left(300 \mathrm{MHz}, \mathrm{DMSO}-d_{6}\right) \delta 4.37$ (s, 2H, $\left.\mathrm{CH}_{2}-\mathrm{CO}\right), 7.73-7.81(\mathrm{~m}$, $3 \mathrm{H}, \mathrm{ArH}), 12.38$ (bs, 1H, NH).

2-[\{5-(2-Hydroxyphenyl)-[1,3,4]-thiadiazol-2-yl $\}$ imino]1,3-thiazolidin-4-one 3(k): mp 160 - $161{ }^{\circ} \mathrm{C}$; \% Yield: 73; IR $(\mathrm{KBr}) \mathrm{cm}^{-1}$ : $3288(\mathrm{NH}), 1696(\mathrm{C}=\mathrm{O}), 1589(\mathrm{C}=\mathrm{N}) ;{ }^{1} \mathrm{H}$ NMR (300 MHz, DMSO- $\left.d_{6}\right) \delta 3.91$ (s, 2H, $\left.\mathrm{CH}_{2}-\mathrm{CO}\right), 7.41-7.59$ (m, $4 \mathrm{H}, \mathrm{ArH}), 9.43$ (s, 1H, OH), 11.76 (bs, 1H, NH).

2-[\{5-(4-Methoxyphenyl)-[1,3,4]-thiadiazol-2-yl\} imino]1,3-thiazolidin-4-one 3(I): mp $108-110^{\circ} \mathrm{C}$; \%Yield: 82; IR $(\mathrm{KBr}) \mathrm{cm}^{-1}$ : $3428(\mathrm{NH}), 1735(\mathrm{C}=\mathrm{O}), 1591(\mathrm{C}=\mathrm{N}) ;{ }^{1} \mathrm{H}$ NMR $\left(300 \mathrm{MHz}, \mathrm{DMSO}-d_{6}\right) \delta 3.64\left(\mathrm{~s}, 3 \mathrm{H}, \mathrm{OCH}_{3}\right), 4.36\left(\mathrm{~s}, 2 \mathrm{H}, \mathrm{CH}_{2}-\right.$ $\mathrm{CO}), 7.37-7.51$ (m, 4H, ArH), 12.54 (bs, 1H, NH).

General method for synthesis of 5-benzylidene-2-[\{5-(substituted phenyl)-[1,3,4]-thiadiazol-2-yl\} imino]-1,3-thiazolidin4-one 4(a-l). To compound 3(a-l) (3 mmol) in $20 \mathrm{~mL}$ ethanol, $2 \mathrm{~mL}$ piperidine and $3 \mathrm{mmol}$ of benzaldehyde was added. The mixture was refluxed for 12 - $18 \mathrm{hrs}$. The mixture was poured over crushed ice and the solution was neutralized with $\mathrm{HCl}$. The precipitate was filtered, washed with water, dried and recrystallized from ethanol-DMF to yield final compound 4(a-l). The purity of compounds was analyzed by TLC using benzene: acetone $(9: 1)$ as mobile phase.

5-Benzylidene-2-[\{5-phenyl-[1,3,4]-thiadiazol-2-yl\} imino]1,3-thiazolidin-4-one 4(a): $\mathrm{mp} 88-90{ }^{\circ} \mathrm{C}$; \% Yield: 73; IR (KBr) $\mathrm{cm}^{-1}$ : $3328(\mathrm{NH}), 1758(\mathrm{C}=\mathrm{O}), 1593(\mathrm{C}=\mathrm{N}) ;{ }^{1} \mathrm{H}$ NMR $(300$ $\left.\mathrm{MHz}, \mathrm{DMSO}-d_{6}\right) \delta$ 7.21-7.53 (m, 1H-CH, 10H-ArH), 12.06 (bs, $1 \mathrm{H}, \mathrm{NH}, \mathrm{D}_{2} \mathrm{O}$-exchangeble); ${ }^{13} \mathrm{C}$ NMR $\left(75 \mathrm{MHz}, \mathrm{DMSO}-d_{6}\right) \delta$ $120.7,125.9,126.4,126.8,127.1,127.4,129.4,130.3,136.5$, $142.5,154.3,162.9,163.4,164.2,171.7$; FAB-MS m/z: 364 $(\mathrm{M})^{+}$

5-Benzylidene-2-[\{5-(2-methylphenyl)-[1,3,4]-thiadiazol2-yl\} imino]-1,3-thiazolidin-4-one 4(b): mp 114 - $116{ }^{\circ} \mathrm{C} ; \%$ Yield: 71; IR (KBr) cm ${ }^{-1}$ : $3296(\mathrm{NH}), 1769(\mathrm{C}=\mathrm{O}), 1571(\mathrm{C}=\mathrm{N})$; ${ }^{1} \mathrm{H}$ NMR $\left(300 \mathrm{MHz}, \mathrm{DMSO}-d_{6}\right) \delta 2.33\left(\mathrm{~s}, 3 \mathrm{H}, \mathrm{CH}_{3}\right), 7.10-7.36$ (m, 9H, ArH), 7.42 (s, 1H, CH), 11.79 (bs, 1H, NH).

5-Benzylidene-2-[\{5-(4-methylphenyl)-[1,3,4]-thiadiazol- 2-yl\} iminol-1,3-thiazolidin-4-one 4(c): mp 138 - $139{ }^{\circ} \mathrm{C} ; \%$ Yield: 69; IR (KBr) cm ${ }^{-1}$ : $3307(\mathrm{NH}), 1710(\mathrm{C}=\mathrm{O}), 1599(\mathrm{C}=\mathrm{N})$; ${ }^{1} \mathrm{H}$ NMR $\left(300 \mathrm{MHz}, \mathrm{DMSO}-d_{6}\right) \delta 2.35\left(\mathrm{~s}, 3 \mathrm{H}, \mathrm{CH}_{3}\right), 7.12-7.38$ (m, 9H, ArH), 7.46 (s, 1H, CH), 11.69 (bs, 1H, NH).

5-Benzylidene-2-[\{5-(2-chlorophenyl)-[1,3,4]-thiadiazol2-yl\} imino]-1,3-thiazolidin-4-one 4(d): mp 122 - $124{ }^{\circ} \mathrm{C} ; \%$ Yield: 65; IR (KBr) cm ${ }^{-1}: 3357(\mathrm{NH}), 1754(\mathrm{C}=\mathrm{O}), 1597(\mathrm{C}=\mathrm{N})$; ${ }^{1} \mathrm{H}$ NMR $\left(300 \mathrm{MHz}, \mathrm{DMSO}-d_{6}\right) \delta$ 7.14-7.48 (m, 1H-CH, 9HArH), 12.19 (bs, 1H, NH).

5-Benzylidene-2-[\{5-(4-chlorophenyl)-[1,3,4]-thiadiazol2-yl imino]-1,3-thiazolidin-4-one 4(e): mp 147 - $149{ }^{\circ} \mathrm{C} ; \%$ Yield: 78; IR (KBr) cm ${ }^{-1}$ : $3328(\mathrm{NH}), 1714(\mathrm{C}=\mathrm{O}), 1564(\mathrm{C}=\mathrm{N})$; ${ }^{1} \mathrm{H}$ NMR $\left(300 \mathrm{MHz}, \mathrm{DMSO}-d_{6}\right) \delta 7.16-7.52$ (m, $1 \mathrm{H}-\mathrm{CH}, 9 \mathrm{H}-$ ArH), 12.31 (bs, 1H, NH).

5-Benzylidene-2-[\{5-(2-bromophenyl)-[1,3,4]-thiadiazol2-yl\} imino]-1,3-thiazolidin-4-one 4(f): mp $108-109{ }^{\circ} \mathrm{C} ; \%$ Yield: 61; IR (KBr) cm ${ }^{-1}$ : $3271(\mathrm{NH}), 1726(\mathrm{C}=\mathrm{O}), 1542(\mathrm{C}=\mathrm{N})$; ${ }^{1} \mathrm{H}$ NMR $\left(300 \mathrm{MHz}, \mathrm{DMSO}-d_{6}\right) \delta$ 7.14-7.49 (m, 1H-CH, 9HArH), 11.66 (bs, 1H, NH).

5-Benzylidene-2-[\{5-(4-bromophenyl)-[1,3,4]-thiadiazol2-yl\} iminol-1,3-thiazolidin-4-one 4(g): mp 83 - $84{ }^{\circ} \mathrm{C}$; \% Yield: 68; IR (KBr) cm ${ }^{-1}$ : $3313(\mathrm{NH}), 1780(\mathrm{C}=\mathrm{O}), 1577(\mathrm{C}=\mathrm{N}) ;{ }^{1} \mathrm{H}$ NMR (300 MHz, DMSO-d $) \delta$ 7.20-7.58 (m, 1H-CH, 9H-ArH), 12.24 (bs, $1 \mathrm{H}, \mathrm{NH}, \mathrm{D}_{2} \mathrm{O}$-exchangeble); ${ }^{13} \mathrm{C}$ NMR $(75 \mathrm{MHz}$, DMSO-d $) \delta 120.7,125.9,126.4,126.8,127.1,127.4,129.4$, $130.3,136.5,142.5,154.3,162.9,163.4,164.2$, 171.7; FAB-MS $m / z: 443(\mathrm{M})^{+}, 445(\mathrm{M}+2)^{+}$

5-Benzylidene-2-[\{5-(3-nitrophenyl)-[1,3,4]-thiadiazol-2yl\} iminol-1,3-thiazolidin-4-one 4(h): mp $109-111^{\circ} \mathrm{C}$; \%Yield: 57; IR (KBr) cm ${ }^{-1}$ : $3374(\mathrm{NH}), 1737(\mathrm{C}=\mathrm{O}), 1568(\mathrm{C}=\mathrm{N}) ;{ }^{1} \mathrm{H}$ NMR (300 MHz, DMSO- $\left.d_{6}\right) \delta$ 6.69-7.30 (m, 9H, ArH), 7.37 (s, 1H, CH), 11.26 (bs, 1H, NH).

5-Benzylidene-2-[\{5-(4-nitrophenyl)-[1,3,4]-thiadiazol-2yl imino]-1,3-thiazolidin-4-one 4(i): $\mathrm{mp} 132-135{ }^{\circ} \mathrm{C}$; \% Yield: 53; IR (KBr) cm ${ }^{-1}$ : $3286(\mathrm{NH}), 1694(\mathrm{C}=\mathrm{O}), 1585(\mathrm{C}=\mathrm{N}) ;{ }^{1} \mathrm{H}$ NMR (300 MHz, DMSO- $\left.d_{6}\right) \delta$ 6.79-7.32 (m, 9H, ArH), 7.39 (s, $1 \mathrm{H}, \mathrm{CH}), 10.98$ (bs, 1H, NH).

5-Benzylidene-2-[\{5-(2,4-dichlorophenyl)-[1,3,4]-thiadiazol-2-yl\} imino]-1,3-thiazolidin-4-one 4(j): mp 116 - $117^{\circ} \mathrm{C} ; \%$ Yield: 63; IR (KBr) cm ${ }^{-1}$ : $3358(\mathrm{NH}), 1729(\mathrm{C}=\mathrm{O}), 1547(\mathrm{C}=\mathrm{N})$; ${ }^{1} \mathrm{H}$ NMR $\left(300 \mathrm{MHz}, \mathrm{DMSO}-d_{6}\right) \delta$ 7.24-7.51 (m, $1 \mathrm{H}-\mathrm{CH}, 8 \mathrm{H}-$ ArH), 11.41 (bs, 1H, NH).

5-Benzylidene-2-[\{5-(2-hydroxyphenyl)-[1,3,4]-thiadiazol2-yl\} imino]-1,3-thiazolidin-4-one 4(k): mp 90 - $93{ }^{\circ} \mathrm{C}$; \% Yield: 79; IR (KBr) cm ${ }^{-1}: 3451(\mathrm{OH}), 3225(\mathrm{NH}), 1658(\mathrm{C}=\mathrm{O}), 1592$ $(\mathrm{C}=\mathrm{N}) ;{ }^{1} \mathrm{H}$ NMR $\left(300 \mathrm{MHz}, \mathrm{DMSO}-d_{6}\right) \delta 6.96-7.37(\mathrm{~m}, 1 \mathrm{H}-\mathrm{CH}$, 9H-ArH), 9.41 (s1H, OH), 11.29 (bs, 1H, NH).

5-Benzylidene-2-[\{5-(4-methoxyphenyl)-[1,3,4]-thiadiazol-2-yl\} imino]-1,3-thiazolidin-4-one 4(I): $\mathrm{mp} 98$ - $100{ }^{\circ} \mathrm{C}$; \% Yield: 67; IR (KBr) cm ${ }^{-1}$ : $3347(\mathrm{NH}), 1722(\mathrm{C}=\mathrm{O}), 1573(\mathrm{C}=\mathrm{N})$; ${ }^{1} \mathrm{H}$ NMR $\left(300 \mathrm{MHz}, \mathrm{DMSO}-d_{6}\right) \delta 3.74\left(\mathrm{~s}, 3 \mathrm{H}, \mathrm{OCH}_{3}\right), 6.84-$ 7.53 (m, 1H-CH, 9H-ArH), 11.88 (bs, 1H, NH).

General method for synthesis of 2 -[ $\{5$-(substituted phenyl)[1,3,4]-thiadiazol-2-yl\} imino]-5-(3,4-dimethoxybenzylidene)1,3-thiazolidin-4-one 5(a-l). To the compound 3 (a-l) (3 mmol) in $20 \mathrm{~mL}$ ethanol, $2 \mathrm{~mL}$ piperidine and $3 \mathrm{mmol}$ of 3, 4-dimethoxy benzaldehyde was added. The mixture was refluxed for 12 - 18 hrs. The mixture was poured over crushed ice and the solution 
was neutralized with $\mathrm{HCl}$. The precipitate was filtered, washed with water, dried and recrystallized from ethanol-DMF to yield final compound 5(a-I). The purity of compound was analyzed by TLC using benzene: acetone $(9: 1)$ as mobile phase.

2-[\{5-Phenyl-[1,3,4]-thiadiazol-2-yl $\}$ imino]-5-(3,4-dimethoxybenzylidene)-1,3-thiazolidin-4-one 5(a): $\mathrm{mp} 113$ - $115^{\circ} \mathrm{C}$; \%Yield: 68; IR (KBr) cm ${ }^{-1}$ : $3352(\mathrm{NH}), 1743(\mathrm{C}=\mathrm{O}), 1546$ $(\mathrm{C}=\mathrm{N}) ;{ }^{1} \mathrm{H} \mathrm{NMR}\left(300 \mathrm{MHz}, \mathrm{DMSO}-d_{6}\right) \delta 3.86\left(\mathrm{~s}, 6 \mathrm{H}, 2 \mathrm{xOCH}_{3}\right)$, 6.84-7.93 (m, 1H-CH, 8H-ArH), 12.86 (bs, 1H, NH, D 2 O-exchangeble); ${ }^{13} \mathrm{CNMR}\left(75 \mathrm{MHz}, \mathrm{DMSO}-d_{6}\right) \delta 56.3,115.4,112.4$, 119.6, 126.8, 127.1, 128.4, 129.4, 136.5, 142.5, 143.5, 146.3, 154.2, 163.7, 164.2, 172.7; FAB-MS $m / z: 424(\mathrm{M})^{+}, 425(\mathrm{M}+1)^{+}$.

2-[\{5-(2-Methylphenyl)-[1,3,4]-thiadiazol-2-yl $\}$ imino]-5(3,4-dimethoxybenzylidene)-1,3-thiazolidin-4-one 5(b): $\mathrm{mp}$ 96 - $99^{\circ} \mathrm{C}$; \% Yield: 74; IR (KBr) cm $\mathrm{cm}^{-1}: 3330(\mathrm{NH}), 1738(\mathrm{C}=\mathrm{O})$, $1556(\mathrm{C}=\mathrm{N}) ;{ }^{1} \mathrm{H}$ NMR $\left(300 \mathrm{MHz}, \mathrm{DMSO}-d_{6}\right) \delta 2.35(\mathrm{~s}, 3 \mathrm{H}$, $\left.\mathrm{CH}_{3}\right), 3.73$ (s, 6H, 2xOCH 3 ), 7.26-8.20 (m, 1H-CH, 7H-ArH), 11.68 (bs, $1 \mathrm{H}, \mathrm{NH})$.

2-[\{5-(4-Methylphenyl)-[1,3,4]-thiadiazol-2-yl\} imino]-5(3,4-dimethoxybenzylidene)-1,3-thiazolidin-4-one 5(c): $\mathrm{mp}$ 91 - $93{ }^{\circ} \mathrm{C}$; \% Yield: 59; IR ( $\left.\mathrm{KBr}\right) \mathrm{cm}^{-1}: 3287(\mathrm{NH}), 1699(\mathrm{C}=\mathrm{O})$, $1523(\mathrm{C}=\mathrm{N}) ;{ }^{1} \mathrm{H}$ NMR $\left(300 \mathrm{MHz}, \mathrm{DMSO}-d_{6}\right) \delta 2.32(\mathrm{~s}, 3 \mathrm{H}$, $\mathrm{CH}_{3}$ ), 3.81 (s, 6H, 2xOCH 3 ), 7.16-7.98 (m, 1H-CH, 7H-ArH), 12.34 (bs, $1 \mathrm{H}, \mathrm{NH})$.

2-[\{5-(2-Chlorophenyl)-[1,3,4]-thiadiazol-2-yl $\}$ imino $]-5-$ (3,4-dimethoxybenzylidene)-1,3-thiazolidin-4-one 5(d): $\mathrm{mp}$ 110 - $112{ }^{\circ} \mathrm{C}$; \%Yield: 64; IR (KBr) cm ${ }^{-1}$ : $3316(\mathrm{NH}), 1777$ $(\mathrm{C}=\mathrm{O}), 1525(\mathrm{C}=\mathrm{N}) ;{ }^{1} \mathrm{H}$ NMR $\left(300 \mathrm{MHz}, \mathrm{DMSO}-d_{6}\right) \delta 3.58$ (s, $\left.6 \mathrm{H}, 2 \mathrm{xOCH}_{3}\right), 7.37-8.06$ (m, 1H-CH, 7H-ArH), 10.44 (bs, $1 \mathrm{H}, \mathrm{NH})$.

2-[\{5-(4-Chlorophenyl)-[1,3,4]-thiadiazol-2-yl $\}$ imino $]-5-$ (3,4-dimethoxybenzylidene)-1,3-thiazolidin-4-one 5(e): $\mathrm{mp}$ 104 - $106{ }^{\circ} \mathrm{C}$; \%Yield: 72; IR (KBr) cm ${ }^{-1}: 3288(\mathrm{NH}), 1762$ $(\mathrm{C}=\mathrm{O}), 1541(\mathrm{C}=\mathrm{N}) ;{ }^{1} \mathrm{H}$ NMR $\left(300 \mathrm{MHz}, \mathrm{DMSO}-d_{6}\right) \delta 3.64(\mathrm{~s}$, $6 \mathrm{H}, 2 \mathrm{xOCH}_{3}$ ), 7.41-8.18 (m, 1H-CH, 7H-ArH), 12.04 (bs, 1H, $\mathrm{NH})$.

2-[\{5-(2-Bromophenyl)-[1,3,4]-thiadiazol-2-yl\} imino]-5(3,4-dimethoxybenzylidene)-1,3-thiazolidin-4-one 5(f): $\mathrm{mp}$ 85 - $87^{\circ} \mathrm{C}$; \% Yield: 76; IR (KBr) cm $\mathrm{cm}^{-1}: 3328(\mathrm{NH}), 1728(\mathrm{C}=\mathrm{O})$, $1553(\mathrm{C}=\mathrm{N}) ;{ }^{1} \mathrm{H}$ NMR $\left(300 \mathrm{MHz}, \mathrm{DMSO}-d_{6}\right) \delta 3.42(\mathrm{~s}, 6 \mathrm{H}$, $\left.2 \mathrm{xOCH}_{3}\right), 7.22-7.89$ (m, 1H-CH, 7H-ArH), 11.62 (bs, 1H, NH).

2-[\{5-(4-Bromophenyl)-[1,3,4]-thiadiazol-2-yl $\}$ imino]-5(3,4-dimethoxybenzylidene)-1,3-thiazolidin-4-one $\mathbf{5}(\mathrm{g})$ : $\mathrm{mp}$ 79 - $81{ }^{\circ} \mathrm{C}$; \% Yield: 84; IR (KBr) cm ${ }^{-1}: 3280(\mathrm{NH}), 1698(\mathrm{C}=\mathrm{O})$, $1518(\mathrm{C}=\mathrm{N}) ;{ }^{1} \mathrm{H}$ NMR $\left(300 \mathrm{MHz}, \mathrm{DMSO}-d_{6}\right) \delta 3.79(\mathrm{~s}, 6 \mathrm{H}$, $2 \mathrm{xOCH}_{3}$ ), 7.28-7.99 (m, 1H-CH, 7H-ArH), 11.39 (bs, $1 \mathrm{H}, \mathrm{NH}$, $\mathrm{D}_{2} \mathrm{O}$-exchangeble).

2-[\{5-(3-Nitrophenyl)-[1,3,4]-thiadiazol-2-yl $\}$ imino]-5(3,4-dimethoxybenzylidene)-1,3-thiazolidin-4-one 5(h): $\mathrm{mp}$ 85 - $86{ }^{\circ} \mathrm{C}$; \% Yield: 77; IR (KBr) cm ${ }^{-1}: 3298(\mathrm{NH}), 1767(\mathrm{C}=\mathrm{O})$, $1561(\mathrm{C}=\mathrm{N}) ;{ }^{1} \mathrm{H}$ NMR $\left(300 \mathrm{MHz}, \mathrm{DMSO}-d_{6}\right) \delta 3.82(\mathrm{~s}, 6 \mathrm{H}$, $\left.2 \mathrm{xOCH}_{3}\right), 7.39-8.62$ (m, 1H-CH, 7H-ArH), 10.84 (bs, 1H, NH).

2-[\{5-(4-Nitrophenyl)-[1,3,4]-thiadiazol-2-yl $\}$ imino]-5(3,4-dimethoxybenzylidene)-1,3-thiazolidin-4-one 5(i): $\mathrm{mp}$ 94 - $96{ }^{\circ} \mathrm{C}$; \% Yield: 67; IR ( $\left.\mathrm{KBr}\right) \mathrm{cm}^{-1}: 3284(\mathrm{NH}), 1700(\mathrm{C}=\mathrm{O})$, $1553(\mathrm{C}=\mathrm{N}) ;{ }^{1} \mathrm{H}$ NMR $\left(300 \mathrm{MHz}, \mathrm{DMSO}-d_{6}\right) \delta 3.86(\mathrm{~s}, 6 \mathrm{H}$, $\left.2 \mathrm{xOCH}_{3}\right), 7.27-7.86$ (m, 1H-CH, 7H-ArH), 10.21 (bs, 1H, NH).

2-[\{5-(2,4-Dichlorophenyl)-[1,3,4]-thiadiazol-2-yl $\}$ imino]- 5-(3,4-dimethoxybenzylidene)-1,3-thiazolidin-4-one 5(j): $\mathrm{mp}$ 117 - $120{ }^{\circ} \mathrm{C}$; \%Yield: 71; IR (KBr) cm $\mathrm{cm}^{-1}: 3348(\mathrm{NH}), 1717$ $(\mathrm{C}=\mathrm{O}), 1542(\mathrm{C}=\mathrm{N}) ;{ }^{1} \mathrm{H}$ NMR $\left(300 \mathrm{MHz}, \mathrm{DMSO}-d_{6}\right) \delta 3.51(\mathrm{~s}$, $6 \mathrm{H}, 2 \mathrm{xOCH}_{3}$ ), 7.42-8.28 (m, 1H-CH, 6H-ArH), 11.64 (bs, 1H, $\mathrm{NH})$.

2-[\{5-(2-Hydroxyphenyl)-[1,3,4]-thiadiazol-2-yl $\}$ imino]5-(3,4-dimethoxybenzylidene)-1,3-thiazolidin-4-one 5(k): mp 79 - $80{ }^{\circ} \mathrm{C}$; \% Yield: 82; IR (KBr) cm ${ }^{-1}: 3418(\mathrm{OH}), 3309(\mathrm{NH})$, $1726(\mathrm{C}=\mathrm{O}), 1565(\mathrm{C}=\mathrm{N}) ;{ }^{1} \mathrm{H}$ NMR $\left(300 \mathrm{MHz}, \mathrm{DMSO}-d_{6}\right) \delta$ $3.23\left(\mathrm{~s}, 6 \mathrm{H}, 2 \mathrm{xOCH}_{3}\right), 7.22-7.98(\mathrm{~m}, 1 \mathrm{H}-\mathrm{CH}, 7 \mathrm{H}-\mathrm{ArH}), 9.43$ (s, $1 \mathrm{H}, \mathrm{OH}), 12.15$ (bs, 1H, NH).

2-[\{5-(4-Methoxyphenyl)-[1,3,4]-thiadiazol-2-yl\} imino]5-(3,4-dimethoxybenzylidene)-1,3-thiazolidin-4-one 5(l): $\mathrm{mp}$ 157 - $158{ }^{\circ} \mathrm{C}$; \% Yield: 54; IR (KBr) cm ${ }^{-1}: 3322(\mathrm{NH}), 1678$ $(\mathrm{C}=\mathrm{O}), 1524(\mathrm{C}=\mathrm{N}) ;{ }^{1} \mathrm{H}$ NMR $\left(300 \mathrm{MHz}, \mathrm{DMSO}-d_{6}\right) \delta 3.61(\mathrm{~s}$, $9 \mathrm{H}, 3 \mathrm{xOCH}_{3}$ ), 7.19-7.63 (m, 1H-CH, 7H-ArH), 10.92 (bs, 1H, $\mathrm{NH})$.

\section{Result and Discussion}

Twenty-four 5-(substituted arylidene)-2-[5-(substituted phenyl)-(1,3,4)thiadiazol-2-yl imino] thiazolidin-4-one [4\&5(a-l)] containing certain groups as substituents on the phenyl ring along with benzylidene or 3,4-dimethoxy substituted benzylidene ring were synthesized by the knoevenagel condensation at position 5 of thiazolidin-4-one moiety with benzaldehyde and 3,4-dimethoxy benzaldehyde in an intermediate compound 3(a-l).

Various substituted benzoic acids were initially treated with thiosemicarbazide in presence of cyclizing agent $\mathrm{POCl}_{3}$ to give compounds $\mathbf{1}(\mathrm{a}-\mathrm{I}){ }^{14}$ The formation of the intermediate was confirmed on the basis of their IR and ${ }^{1} \mathrm{H}$ NMR data.

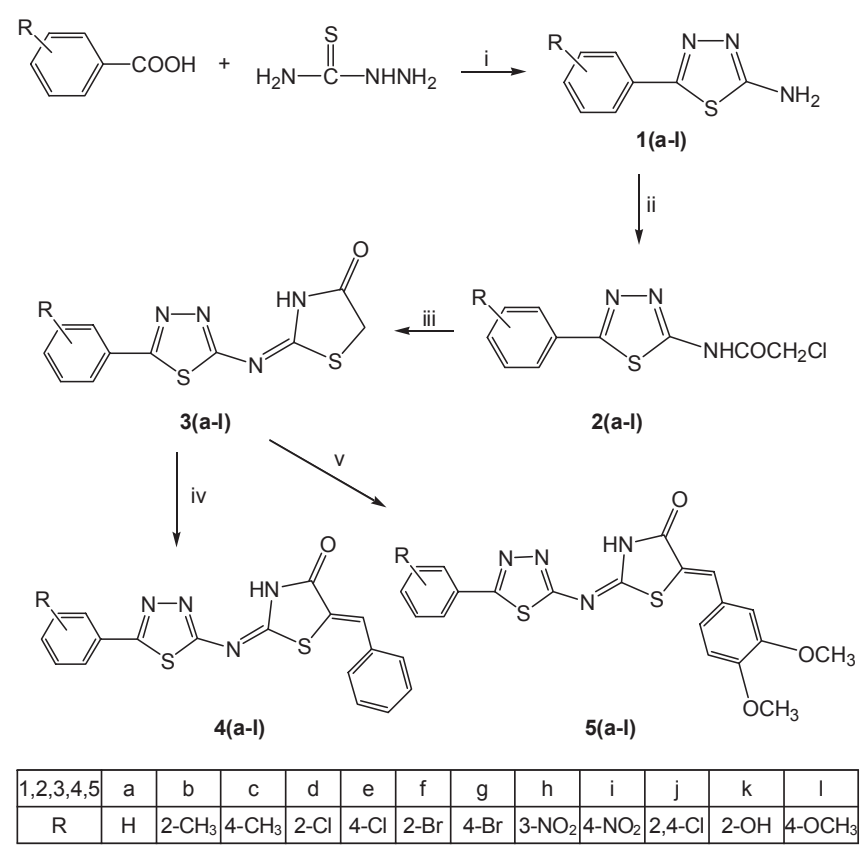

Scheme 1. (i) $\mathrm{POCl}_{3}$, reflux; (ii) $\mathrm{ClCOCH}_{2} \mathrm{Cl}$, dry pyridine, dry benzene, reflux; (iii) $\mathrm{NH}_{4} \mathrm{SCN}$, ethanol, reflux; (iv) benzaldehyde, piperidine, absolute ethanol; (v) 3, 4-dimethoxy benzaldehyde, piperidine, absolute ethanol. 
Table 1. Antimicrobial activity for synthesized compounds

\begin{tabular}{|c|c|c|c|c|c|c|c|c|c|c|}
\hline \multirow{2}{*}{ Comp. } & \multirow{2}{*}{$\begin{array}{l}\text { Conc. } \\
(\mu \mathrm{g} / \mathrm{mL})\end{array}$} & \multicolumn{9}{|c|}{ Zone of inhibition $(\mathrm{mm}) \pm \mathrm{SD}$} \\
\hline & & A & B & $\mathrm{C}$ & $\mathrm{D}$ & $\mathrm{E}$ & $\mathrm{F}$ & G & $\mathrm{H}$ & I \\
\hline \multirow{2}{*}{$4 a$} & 200 & $11.00 \pm 0.40$ & $12.62 \pm 0.21$ & $12.33 \pm 0.51$ & $x$ & $16.84 \pm 0.44$ & $13.67 \pm 0.35$ & $12.38 \pm 0.27$ & $11.60 \pm 0.27$ & $16.79 \pm 0.42$ \\
\hline & 100 & $6.73 \pm 0.61$ & $7.58 \pm 0.36$ & $7.29 \pm 0.42$ & $x$ & $11.86 \pm 0.27$ & $10.33 \pm 0.29$ & $6.83 \pm 0.51$ & $9.25 \pm 0.33$ & $8.37 \pm 0.26$ \\
\hline \multirow{2}{*}{$4 c$} & 200 & $9.85 \pm 0.28$ & $11.02 \pm 0.23$ & $10.93 \pm 0.26$ & $x$ & $11.64 \pm 0.21$ & $9.44 \pm 0.69$ & $10.71 \pm 0.42$ & $10.60 \pm 0.20$ & $11.76 \pm 0.26$ \\
\hline & 100 & $5.73 \pm 0.54$ & $6.63 \pm 0.27$ & $6.28 \pm 0.37$ & $x$ & $6.76 \pm 0.31$ & $5.23 \pm 0.38$ & $6.04 \pm 0.33$ & $5.23 \pm 0.41$ & $6.96 \pm 0.38$ \\
\hline \multirow{2}{*}{$4 d$} & 200 & $15.87 \pm 0.52$ & $18.68 \pm 0.44$ & $16.05 \pm 0.42$ & $x$ & $19.40 \pm 0.42$ & $15.28 \pm 0.51$ & $14.77 \pm 0.21$ & $13.24 \pm 0.42$ & $18.35 \pm 0.27$ \\
\hline & 100 & $10.84 \pm 0.71$ & $11.67 \pm 0.34$ & $10.57 \pm 0.19$ & $x$ & $12.55 \pm 0.24$ & $9.68 \pm 0.73$ & $7.74 \pm 0.23$ & $6.99 \pm 0.20$ & $12.53 \pm 0.33$ \\
\hline \multirow{2}{*}{$4 \mathrm{e}$} & 200 & $16.33 \pm 0.29$ & $20.48 \pm 0.44$ & $18.32 \pm 0.38$ & $x$ & $19.07 \pm 0.28$ & $17.35 \pm 0.31$ & $17.32 \pm 0.22$ & $17.35 \pm 0.28$ & $18.74 \pm 0.24$ \\
\hline & 100 & $10.60 \pm 0.41$ & $13.58 \pm 0.29$ & $11.80 \pm 0.46$ & $x$ & $14.61 \pm 0.31$ & $13.63 \pm 0.45$ & $13.61 \pm 0.27$ & $13.98 \pm 0.29$ & $14.74 \pm 0.33$ \\
\hline \multirow{2}{*}{$4 \mathrm{i}$} & 200 & $13.67 \pm 0.38$ & $14.62 \pm 0.26$ & $14.50 \pm 0.54$ & $x$ & $18.69 \pm 0.46$ & $15.62 \pm 0.26$ & $14.60 \pm 0.24$ & $14.84 \pm 0.43$ & $17.00 \pm 0.42$ \\
\hline & 100 & $8.43 \pm 0.42$ & $9.27 \pm 0.43$ & $8.75 \pm 0.65$ & $x$ & $13.53 \pm 0.25$ & $12.59 \pm 0.22$ & $10.87 \pm 0.28$ & $12.52 \pm 0.31$ & $12.76 \pm 0.51$ \\
\hline \multirow{2}{*}{$4 j$} & 200 & $17.61 \pm 0.29$ & $20.65 \pm 0.42$ & $18.07 \pm 0.22$ & $10.63 \pm 0.38$ & $19.45 \pm 0.29$ & $18.61 \pm 0.32$ & $17.29 \pm 0.18$ & $18.33 \pm 0.42$ & $19.55 \pm 0.39$ \\
\hline & 100 & $10.49 \pm 0.33$ & $13.05 \pm 0.33$ & $11.66 \pm 0.28$ & $x$ & $15.08 \pm 0.51$ & $14.8 \pm 0.29$ & $13.86 \pm 0.51$ & $14.16 \pm 0.33$ & $15.39 \pm 0.40$ \\
\hline \multirow{2}{*}{$4 \mathrm{k}$} & 200 & $16.36 \pm 0.39$ & $18.68 \pm 0.38$ & $17.62 \pm 0.20$ & $x$ & $18.99 \pm 0.42$ & $16.76 \pm 0.27$ & $16.52 \pm 0.22$ & $16.59 \pm 0.26$ & $18.23 \pm 0.26$ \\
\hline & 100 & $9.94 \pm 0.46$ & $12.67 \pm 0.25$ & $11.73 \pm 0.26$ & $x$ & $14.01 \pm 0.42$ & $13.21 \pm 0.51$ & $12.50 \pm 0.69$ & $13.63 \pm 0.27$ & $13.57 \pm 0.29$ \\
\hline \multirow{2}{*}{$5 \mathrm{a}$} & 200 & $14.22 \pm 0.37$ & $18.12 \pm 0.39$ & $15.46 \pm 0.20$ & $x$ & $18.71 \pm 0.20$ & $15.44 \pm 0.23$ & $13.67 \pm 0.26$ & $15.72 \pm 0.54$ & $18.27 \pm 0.27$ \\
\hline & 100 & $9.76 \pm 0.42$ & $12.50 \pm 0.39$ & $11.66 \pm 0.31$ & $x$ & $13.85 \pm 0.31$ & $11.53 \pm 0.46$ & $8.41 \pm 0.33$ & $11.49 \pm 0.20$ & $12.96 \pm 0.35$ \\
\hline \multirow{2}{*}{$5 b$} & 200 & $11.13 \pm 0.54$ & $12.83 \pm 0.27$ & $11.25 \pm 0.34$ & $x$ & $13.29 \pm 0.35$ & $10.92 \pm 0.23$ & $10.52 \pm 0.26$ & $11.42 \pm 0.65$ & $13.75 \pm 0.49$ \\
\hline & 100 & $6.13 \pm 1.16$ & $7.51 \pm 0.41$ & $6.86 \pm 0.29$ & $x$ & $8.64 \pm 0.21$ & $6.50 \pm 0.51$ & $5.78 \pm 0.29$ & $6.76 \pm 0.29$ & $8.57 \pm 0.57$ \\
\hline \multirow{2}{*}{$5 \mathrm{c}$} & 200 & $13.31 \pm 0.41$ & $15.47 \pm 0.22$ & $13.50 \pm 0.27$ & $x$ & $14.68 \pm 0.39$ & $12.53 \pm 0.25$ & $11.63 \pm 0.22$ & $12.56 \pm 0.27$ & $14.46 \pm 0.26$ \\
\hline & 100 & $8.38 \pm 0.57$ & $10.46 \pm 0.33$ & $8.61 \pm 0.41$ & $x$ & $9.49 \pm 0.36$ & $7.20 \pm 0.38$ & $6.50 \pm 0.37$ & $7.37 \pm 0.58$ & $9.50 \pm 0.62$ \\
\hline \multirow{2}{*}{$5 e$} & 200 & $18.40 \pm 0.24$ & $18.69 \pm 0.19$ & $17.77 \pm 0.38$ & $11.60 \pm 0.33$ & $19.77 \pm 0.28$ & $19.35 \pm 0.40$ & $18.31 \pm 0.41$ & $18.58 \pm 0.36$ & $19.92 \pm 0.31$ \\
\hline & 100 & $12.76 \pm 0.27$ & $14.29 \pm 0.52$ & $13.41 \pm 0.36$ & $x$ & $15.23 \pm 0.20$ & $14.84 \pm 0.28$ & $14.38 \pm 0.33$ & $14.68 \pm 0.38$ & $15.58 \pm 0.42$ \\
\hline \multirow{2}{*}{$5 \mathrm{i}$} & 200 & $14.20 \pm 0.59$ & $17.51 \pm 0.43$ & $15.56 \pm 0.29$ & $x$ & $15.48 \pm 0.29$ & $15.53 \pm 0.22$ & $13.56 \pm 0.33$ & $15.32 \pm 0.29$ & $15.49 \pm 0.25$ \\
\hline & 100 & $10.37 \pm 0.27$ & $13.64 \pm 0.37$ & $10.83 \pm 0.25$ & $\times$ & $12.66 \pm 0.38$ & $11.54 \pm 0.36$ & $10.60 \pm 0.22$ & $11.38 \pm 0.34$ & $12.46 \pm 0.37$ \\
\hline \multirow{2}{*}{$5 \mathrm{j}$} & 200 & $18.47 \pm 0.20$ & $19.41 \pm 0.26$ & $18.55 \pm 0.29$ & $12.57 \pm 0.22$ & $19.80 \pm 0.24$ & $19.53 \pm 0.29$ & $17.75 \pm 0.42$ & $18.56 \pm 0.47$ & $19.68 \pm 0.27$ \\
\hline & 100 & $13.30 \pm 0.24$ & $14.57 \pm 0.22$ & $13.62 \pm 0.33$ & $6.35 \pm 0.42$ & $15.43 \pm 0.30$ & $14.81 \pm 0.23$ & $12.43 \pm 0.61$ & $13.53 \pm 0.33$ & $15.25 \pm 0.22$ \\
\hline \multirow{2}{*}{$5 \mathrm{k}$} & 200 & $16.13 \pm 0.38$ & $16.57 \pm 0.21$ & $17.46 \pm 0.40$ & $x$ & $17.67 \pm 0.21$ & $16.44 \pm 0.36$ & $15.97 \pm 0.72$ & $16.49 \pm 0.41$ & $17.65 \pm 0.20$ \\
\hline & 100 & $13.44 \pm 0.18$ & $14.40 \pm 0.30$ & $12.60 \pm 0.28$ & $x$ & $14.58 \pm 0.33$ & $13.19 \pm 023$ & $12.66 \pm 0.54$ & $13.01 \pm 0.38$ & $14.27 \pm 0.42$ \\
\hline \multirow{2}{*}{ Ciprofloxacin } & 200 & $26.52 \pm 0.38$ & $28.31 \pm 0.18$ & $25.83 \pm 0.24$ & $24.58 \pm 0.42$ & NA & NA & NA & NA & NA \\
\hline & 100 & $19.63 \pm 0.21$ & $20.53 \pm 0.42$ & $19.02 \pm 0.28$ & $19.35 \pm 0.27$ & NA & NA & NA & NA & NA \\
\hline \multirow{2}{*}{ Fluconazole } & 200 & NA & NA & NA & NA & $26.35 \pm 0.53$ & $27.56 \pm 0.36$ & $24.43 \pm 0.45$ & $25.75 \pm 0.26$ & $27.48 \pm 0.62$ \\
\hline & 100 & NA & NA & NA & NA & $19.74 \pm 0.28$ & $20.63 \pm 0.24$ & $17.84 \pm 0.61$ & $18.85 \pm 0.39$ & $20.84 \pm 0.37$ \\
\hline
\end{tabular}

A: S. aureus, B: E. coli, C: P. aeruginosa, D: S. typhi, E: A. niger, F: A. flavus, G: M. purpureous, H: P. citrinum, I: C. albicans.

The compounds 1(a-l) were then treated with chloroacetylchloride to produce 2-chloro-N-[5-(substituted-phenyl [1,3,4] thiadiazol-2-yl)]-acetamide 2(a-l) and was characterized by an additional singlet of $\mathrm{CH}_{2}$ in ${ }^{1} \mathrm{H}$ NMR spectra. Further, 2(a-l) was reacted with ammoniumthiocyanate to give cyclized compounds $\mathbf{3}(\mathbf{a}-\mathbf{I})^{15}$ and the singlet for cyclized $\mathrm{CH}_{2}$ shows an upfield shift which was then subjected to knoevenagel condensation with benzaldehyde and 3,4 dimethoxybenzaldehyde to yield final compounds as 5-benzylidene 2-[5-(substituted-phenyl [1,3,4]-thiadiazol-2-ylimino) thiazolidin-4-one 4(a-l) and 2-[5(substituted-phenyl [1,3,4]-thiadiazol-2-ylimino)-5-(3,4-dimethoxy benzylidene) thiazolidin-4-one 5(a-l) respectively (Scheme 1).

The compounds were tested in vitro for antibacterial activity against the test organisms Staphylococcus aureus [ATCC25923], Escherichia coli [ATCC-25922], Pseudomonas aeru- ginosa [NCTC-10662] and Salmonella typhi [Ty2] and for antifungal activity against test organisms Aspergillus niger [MTCC281], Aspergillus flavus [MTCC-277], Monascus purpureous [MTCC-369], Penicillium citrinum [NCIM-768] and Candida albicans [MTCC-3017] by cup-plate technique. ${ }^{16}$ Few of the compounds were selected for antimicrobial activity at concentrations of $200 \mu \mathrm{g} / \mathrm{mL}$ and $100 \mu \mathrm{g} / \mathrm{mL}$. The data was compared to the standard ciprofloxacin for bacteria and fluconazole for fungi.

Compounds showed most significant antibacterial activity against test organism Escherichia coli and most significant antifungal activity against test organisms Aspergillus niger and Candida albicans but on other hand non of the thiadiazole derivative showed even fair activity against Salmonella typhi. Among the evaluated compounds, $\mathbf{5} \mathbf{j}$ was found to be the most active against all bacteria and fungi, as it could inhibit the microbial growth 
at concentration of $100 \mu \mathrm{g} / \mathrm{mL}$ with zone of inhibition ranging from $12.43-15.43 \mathrm{~mm}$. When a comparison is made between the compounds $\mathbf{4 j}$ and $\mathbf{4 e}$ or $\mathbf{5 j}$ and $\mathbf{5 e}$, it appears that compounds with more electronegative groups are more active than the compounds having less electronegative substituents on the first phenyl ring. This was further confirmed by comparing the data for compounds $4 \mathbf{a}, 4 \mathbf{c}$ and $4 \mathrm{e}$ or $\mathbf{5 a}, \mathbf{5 c}$ and $\mathbf{5 e}$. These compounds were found to be active in order as $4 e>4 a>4 c$ or $5 e>5 a>5 c$ where, a represented unsubstitution, $\mathbf{c}$ represented presence of para methyl and $\mathbf{e}$ indicates the chloro group at para position. When the comparison was made between $\mathbf{4 d}$ and $\mathbf{4 e}$ or $\mathbf{5 b}$ and $\mathbf{5 c}$ it was observed that the compounds with para substitution are more active against microbes than ortho substitution and when the comparison was made between all the derivatives of 4(a-l) and 5(a-l) it was observed that compounds possessing methoxy groups at second phenyl ring as in $\mathbf{5}(\mathbf{a}-\mathbf{l})$ are more active than unsubstituted $\mathbf{4}(\mathbf{a}-\mathbf{l})$.

Furthermore, when the comparison for the compounds was made between bacteria and fungi it was observed that the different derivatives of thiadiazole-thiazolidinedione found to be more active against fungi than bacteria and among different bacteria as listed, it was observed that the compounds are more active against gram negative bacteria than the gram positive ones while among different fungi compounds found to be potent against both moulds and yeast.

Acknowledgments. The authors acknowledge the financial support of the University Grant Commission, India and Jamia Hamdard for providing laboratory and other basic facilities for carrying out experimental work. They also wish to express their gratitude to Mrs. P. K. Pillai, Head of the Microbiology Department, Majeedia Hospital, New Delhi, India, in connection with anti-microbial tests.

\section{References}

1. (a) Bloom, B. R.; Cohen, M. L.; Donnenberg, M. S.; Walsh, C.; Aderem, A.; Ulevitch, R. J.; Park, S. H.; Bendelac, A.; Seder, R. A.; Hill, A. V. S.; Fraser, C. M.; Eisen, J. A.; Salzberg, S. L. Nature 2000, 406, 759. (b) Cohen, M. L. Nature 2000, 406, 762.

2. Almasirad, A.; Tabatabai, S. A.; Faizi, M.; Kebriaeezadeh, A.; Mehrabi, N.; Dalvandi, A.; Shafiee, A. Bioorg. Med. Chem. Lett. 2002, 10, 2893.

3. Foroumadi, A.; Kargar, Z.; Sakhteman, A.; Sharifzadeh, Z.; Feyzmohammadi, R.; Kazemi, M.; Shafiee, A. Bioorg. Med. Chem. Lett. 2006, 16, 1164.

4. Michael, R.; Stillings, M. R.; Welbourn, A. P.; Walter, D. S. J. Med. Chem. 1986, 29, 2280.

5. Schenone, S. S.; Brullo, C. C.; Bruno, O. O.; Bondavalli, F. F.; Ranise, A. A.; Filippelli, W. W.; Rinaldi, B. B.; Capuano, A. A.; Falcone, G. G. Bioorg. Med. Chem. 2006, 14, 1698.

6. Clerici, F.; Pocar, D.; Guido, M.; Loche, A.; Perlini, V.; Brufani, M. J. Med. Chem. 2001, 44, 931.

7. Kaya, A. B.; Chizhov, N. P.; Chigareva, N. G. Pharm. Chem. Jour. 1978, 12, 66 .

8. Tunçbilek, M.; Altanlar, N. Il Farmaco 1999, 54, 475.

9. Ergene, N.; Capan, G. Il Farmaco 1994, 49, 449.

10. Diurno, M.V.; Mazzoni, O.; Izzo, A. A.; Bolognese, A. Il Farmaco 1997, 52, 237.

11. Verma, A.; Saraf, S. K.; Eur. J. Med. Chem. 2008, 43, 897.

12. Chavan, A. A.; Pai, N. R. Arkivoc 2007, 148.

13. Diurno, M. V.; Mazzoni, O.; Correale, G.; Monterry, I. G.; Il Farmaco 1999, 54, 579.

14. Shafiee, A.; Naimi, E.; Mansobi, P.; Foroumadi, A.; Shekari, M. J. Het. Chem. 1995, 32, 1235.

15. Altıntaş, H.; Ateş, O.; Kocabalkanli, A.; Birteksoz, S.; Otuk, G. Ind. J. Chem. 2005, 44B, 585.

16. (a) Indian Pharmacopoeia 1996, Appendix 9, A-100. (b) Karegoudar, P.; Karthikeyan, M. S.; Prasad, D. J.; Mahalinga, M.; Shivaramholla, B.; Kumari, N. S. Eur. J. Med. Chem. 2008, 43, 261. (c) Skaggs, B. A. A.; Motley, M.; Warnock, D. W.; Morrissor, C. J. J. Clin. Microbial. 2000, 38, 2254. 